\title{
Physiotherapy after coronary artery surgery: are breathing exercises necessary?
}

\author{
SUSAN C JENKINS, SUSAN A SOUTAR, JULIA M LOUKOTA, LORNA C JOHNSON, \\ JOHN MOXHAM
}

From the Departments of Thoracic Medicine and Physiotherapy, King's College Hospital, London

\begin{abstract}
One hundred and ten men undergoing coronary artery bypass grafting took part in a⿳亠丷厂 prospective randomised study comparing three physiotherapy protocols. All patients were taught selff supported huffing and coughing by a physiotherapist and encouraged to move about. This comprisedoo the sole treatment for the 37 control patients (group 3). Additional physiotherapy included breathinges exercises for the 35 patients in group 1 and use of an incentive spirometer for the 38 patients in group 2. Functional residual capacity (FRC) was measured daily at the bedside until the fifth postoperative day and arterial blood gas tensions were measured on the second and fourth postoperative days. After surgery patients developed a severe restrictive ventilatory defect and profound arterial hypoxaemiaco There were no differences between the three groups. Mean FRC on day 2 was 1.90 litres (61\% of the preoperative value), increasing to 2.321 by day $5(76 \%$ of the preoperative value). The mean arteriat oxygen tension was $7 \cdot 37 \mathrm{kPa}$ on day 2 and $8.58 \mathrm{kPa}$ on day 4 . Four patients in group 1 , two in group 2,00 and five in group 3 developed a chest infection. It is concluded that the addition of breathing exercises or incentive spirometry to a regimen of early mobilisation and huffing and coughing confers no extraミ benefit after uncomplicated coronary artery bypass grafting.
\end{abstract}

\section{Introduction}

After coronary artery bypass grafting physiotherapy - consisting of breathing exercises emphasising inspiration, incentive spirometry, ${ }^{\prime}$ techniques to clear bronchial secretions, and early mobilisation-is given with the aim of increasing lung ventilation and preventing chest infections.

So far all studies comparing physiotherapeutic techniques in patients who have had coronary artery bypass grafting have been carried out in the United States or Canada, ${ }^{2-7}$ and with the exception of one study $^{5}$ have included patients undergoing cardiopulmonary bypass for other surgical procedures. The number of patients randomised to a study group has been eight or less in some cases ${ }^{57}$ and the physiotherapy techniques have on occasions differed considerably from those used in Britain.

The present study was undertaken to investigate whether the addition of breathing exercises or incentive spirometry to a regimen of early mobilisation and instruction in huffing and coughing would prove more effective in improving lung function and preventing

Address for reprint requests: Susan C Jenkins, Department of Thoracic Medicine, King's College School of Medicine and Dentistry, London SE5 9PJ.

Accepted 28 April 1989 chest infection in men recovering from coronary artery $\overrightarrow{\vec{P}}$ bypass grafting. This paper describes a randomised 3 study comparing three physiotherapy protocols in $110 \mathrm{~S}$ such men.

\section{Methods}

\section{PATIENTS}

Consecutive white men undergoing elective coronary artery bypass grafting during one year were considered for inclusion in the study. Patients who had previously? had cardiac surgery and those unable to walk theo length of the ward (64 metres) for reasons other than angina were excluded. The study was approved by theo hospital ethics committee and informed written con sent was obtained from all patients.

\section{LUNG FUNCTION MEASUREMENTS}

Patients were first studied in the afternoon during theo 48 hours before surgery. Pulmonary function was measured at the bedside with the patient sitting upright in a chair, as follows:

1 Lung volumes Functional residual capacityo (FRC) was measured by the steady state heliumo dilution method with a portable spirometer (Pulmonet吕 III, Gould Godart Ltd). Three vital capacity (VC)D breaths were taken at the end of the test and the highest value was used in the calculations. 
2 Arterial oxygen $\left(\mathrm{PaO}_{2}\right)$ and carbon dioxide $\left(\mathrm{PaCO}_{2}\right)$ tensions were measured by analysis of arterialised ear lobe capillary blood. ${ }^{8}$

3 Forced vital capacity (FVC), forced expiratory volume in one second $\left(F E V_{1}\right)$ and peak expiratory flow (PEF) were measured with a Respiradyne spirometer. ${ }^{9}$ The highest values from five maximal forced expirations were taken. Patients with an FEV less than $50 \%$ predicted normal or a forced expiratory ratio (FER) less than $60 \%$ were excluded from the study. Stratified randomisation to balance for age and FER was used to allocate patients to one of three groups.

\section{PHYSIOTHERA PY PROTOCOLS}

All patients were seen before operation by a physiotherapist, who explained the need to move about after surgery and to expectorate excess bronchial secretions. Patients were taught huffing (forced expirations with the glottis open), coughing with sternal support and active exercises of the upper and lower limbs. When they were able they sat in a chair and walked on the second postoperative day; stair climbing was introduced on the fourth day. This was the only postoperative physiotherapy for the control patients (group 3).

In addition to this treatment patients in groups 1 and 2 were given a rationale for taking deep breaths (group 1) or for using an incentive spirometer (group 2) and were treated according to the following protocols:

Group 1 Patients had the usual physiotherapy for patients undergoing cardiac surgery, consisting principally of localised (lateral costal and abdominal) breathing exercises. ${ }^{10}$ Three to five consecutive deep breaths were interspersed between periods of quiet breathing. Patients practised the exercises in the sitting or half lying position, but if the physiotherapist thought that it was indicated patients were treated while lying on their side with chest vibration or gentle percussion to encourage expectoration.

Group 2 Patients were taught to use an incentive spirometer (Triflo II, Sherwood Medical Industries) in the sitting or half lying position. Three to five consecutive breaths with the spirometer were interspersed between periods of quiet breathing.

Patients in groups 1 and 2 were instructed to practise self treatment exercises preoperatively and once extubated to take at least 10 deep breaths (group 1) or to use the incentive spirometer at least 10 times (group 2) in each waking hour until the end of the fifth postoperative day.

After surgery (which was performed via a median sternotomy) all patients were seen by a physiotherapist at least twice on days 1 and 2 and at least once daily on days 3-5. At the end of each treatment, instructions to practise taking deep breaths (group 1) or to use the incentive spirometer (group 2) were given. Treatment sessions lasted on average 10-15 minutes for patients in groups 1 and 2 and somewhat less for patients in group 3. Two senior physiotherapists (JML and LCJ) were responsible for coordinating the study and for closely monitoring the practice of the other physiotherapists treating the patients. Medical and nursing personnel were not told which treatment patients were receiving.

Patients were withdrawn from the study for any of the following reasons: more than 24 hours' assisted ventilation; over 48 hours in the intensive care unit; mobilisation contraindicated; clinically important neurological complications; mental confusion.

\section{ASSESSMENT}

FRC and VC were measured at the bedside on the afternoon of the second postoperative day and at the same time each succeeding day. Arterialised ear lobe capillary blood was sampled on days 2 and 4 with the patient breathing room air. Forced vital capacity, $\mathrm{FEV}_{1}$, and PEF were measured on the fourth day. All measurements were made by SCJ or SAS and were not performed in patients unable to sit in a chair, within one hour of eating, or immediately after treatment by the physiotherapist.

The first chest radiograph after extubation was assessed by a radiologist unaware of the patient's treatment group, who allocated a score $(0=$ clear; $1=$ minor collapse at one base; 2 = pronounced collapse or consolidation (or both) at one base; $3=$ bilateral changes). Subsequent films, taken on day 3 or 4 , were reported as improved, unchanged, or deteriorated.

The following were recorded each day:

1 Maximum oral temperature and medication received (amount of analgesia quantified according to a system of scoring ${ }^{11}$ ).

2 Distance walked by the patient, estimated by the number of journeys to the bathroom and lengths of the ward walked.

3 Patient's perception of chest discomfort, recorded, without knowledge of previous scores, on a visual analogue scale going from "no discomfort" to "severe discomfort."

4 Number of self treatment sessions (groups 1 and 2 only) as reported by the patient (one session was 10 deep breaths or 10 breaths with the incentive spirometer).

A diagnosis of chest infection was made if a patient had a temperature of at least $38.5^{\circ} \mathrm{C}$ and radiological evidence of consolidation or pronounced lung collapse.

STATISTICAL ANALYSIS

Intergroup differences were tested by analysis of 
variance, the Kruskal-Wallis analysis of variance being used to test for differences in data that did not show a normal distribution in the KolmogorovSmirnov goodness of fit test. The $\chi^{2}$ test with Yates's correction and Fisher's exact test were used for qualitative data. Paired and independent $t$ tests were used for within group comparisons and analysis of data on subgroups of patients and the Wilcoxon signed rank and Mann-Whitney U tests for parametric and ranked data respectively. The relation between variables was examined by calculating correlation coefficients. Probability values of less than 0.05 were regarded as significant.

\section{Results}

One hundred and forty one patients were considered for the study. Nine patients with an $\mathrm{FEV}_{1}$ less than $50 \%$ predicted or an FER below $60 \%$ were excluded and one patient refused consent. Twenty one patients were withdrawn after surgery: nine, four, and eight patients from groups 1,2 and 3 . The reasons for withdrawal were: ventilation for more than 24 hours or in intensive care unit (or both) more than 48 hours (five patients), mobilisation contraindicated (four patients), neurological complications (two patients), mental confusion (five patients), lack of cooperation? (five patients).

Results from 110 patients were available for analysis. There was no difference between the threeo groups in the patients' characteristics or preoperative lung function (table), though there was a wide range inlung function values. No patient had a $\mathrm{PaCO}_{2}$ above 5.9. $\mathrm{kPa}$ and the minimum $\mathrm{PaO}_{2}$ was $8.5 \mathrm{kPa}$. Obesity was associated with a low FRC and cigarette smoking with? a low $F E V_{1}$. The groups were matched for surgeon $\vec{x}$ operation, details of cardiopulmonary bypass, trans $-\frac{i}{\perp}$ fusion requirements, and duration of postoperativeio ventilation (mean 11.0 (SD 4.1) hours). The internaio mammary artery was used for grafting in 21 patients in ${ }^{+}$ group 1,29 in group 2 , and 27 in group $3(p>0.05)$. 은

\section{POSTOPERATIVE LUNG FUNCTION}

There was a substantial and persistent reduction in lung volumes and in arterial $\mathrm{Po}_{2}$. FRC, forceds expiratory volumes, PEF, and arterial blood gase tensions in the three groups are shown in figures $1-3 \%$ There were no intergroup differences $(p>0 \cdot 3)$. On the

Characteristics of the 110 men completing the study (mean (SD) values except where otherwise specified)

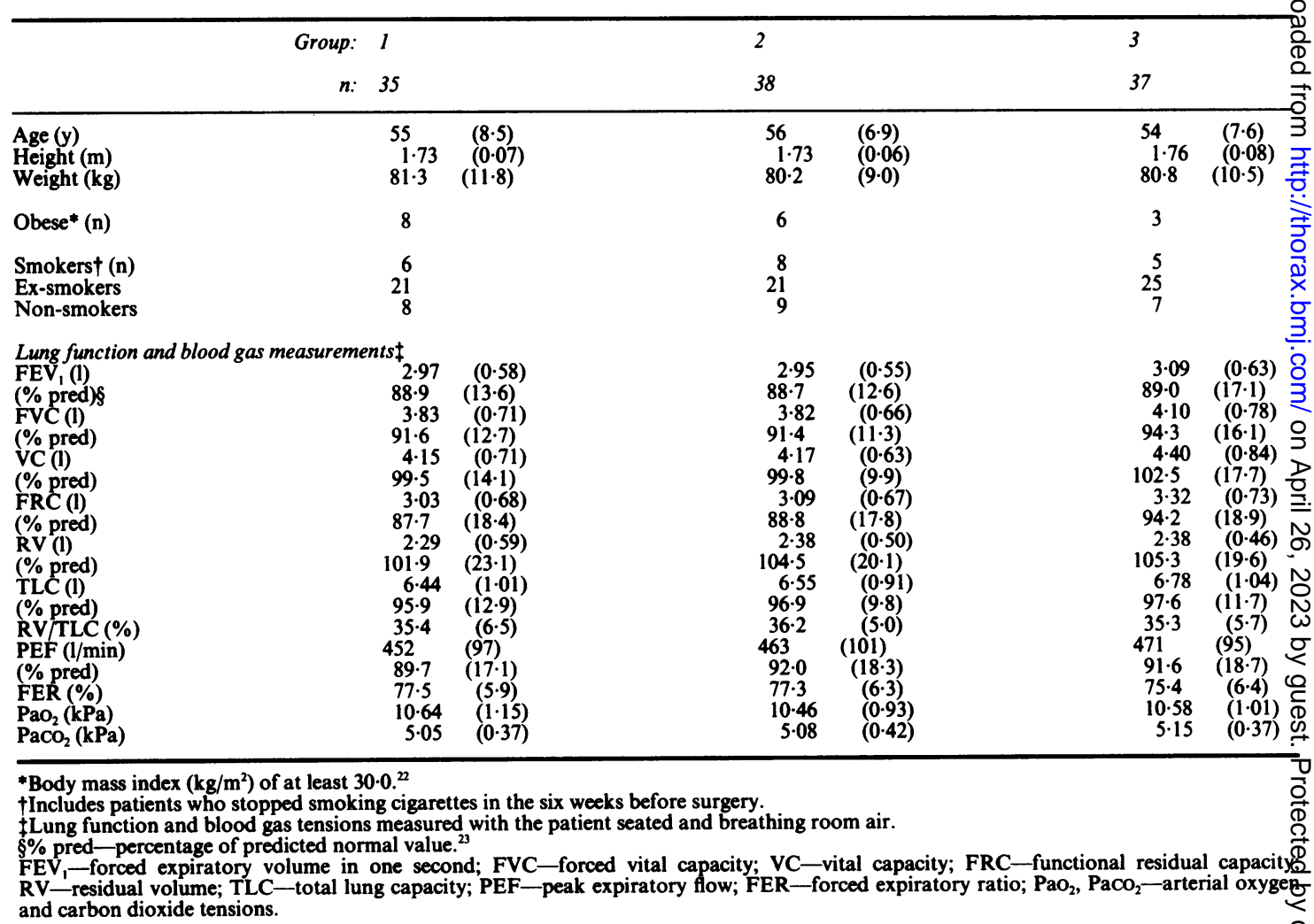

$\mathrm{RV}$-residual volume; TLC-
and carbon dioxide tensions. 
FRC (I)

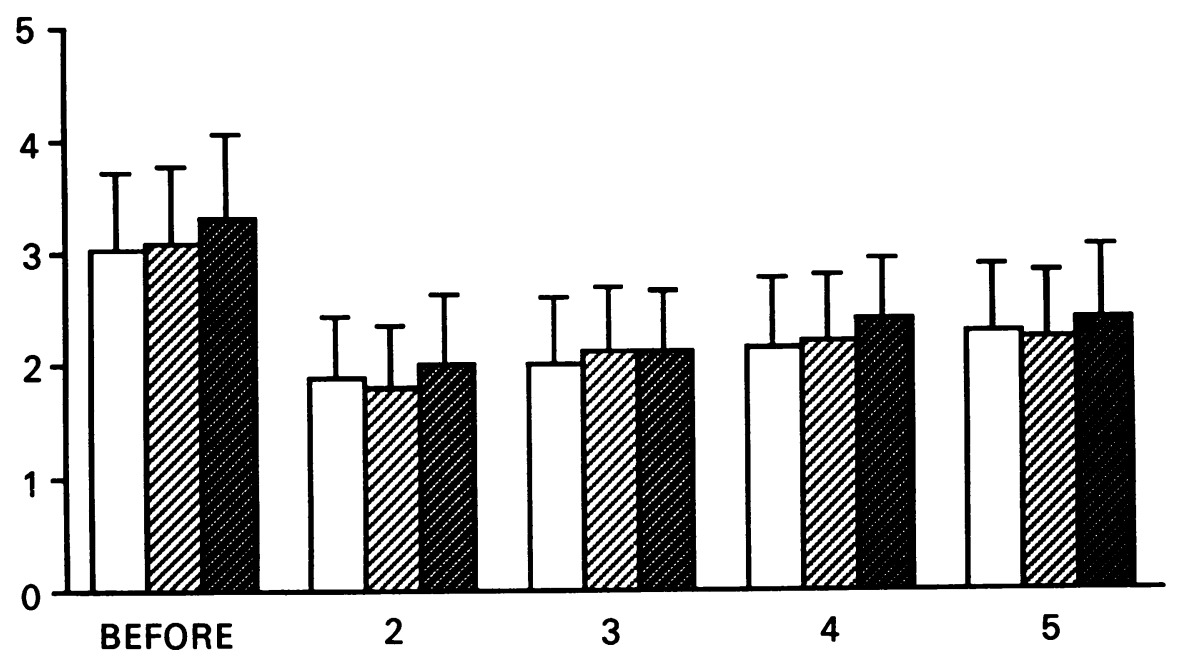

DAYS AFTER OPERATION

Fig 1 Mean ( $S D$ ) functional residual capacity ( $F R C$ ) measured before operation and on days 2, 3,4 , and 5 after coronary artery bypass grafting. The numbers in each group before operation and on days 2, 3, 4, and 5 were: group 1 ( $\square$ ) 35, 28, 35, 34, and 31; group 2 (飞) 38,29,37, 38, and 28; and group 3 () $37,25,35,36$, and 29.

second day mean (SD) FRC was $1.90(0.60)$ litres, VC $1.66(0.53) 1$, and total lung capacity (TLC) $3 \cdot 17(0.83) 1$ $(n=82)$, increasing to $2 \cdot 32(0.65), 2.63(0.63)$, and 4.39 $(0.91) 1$ by day $5(n=88)$. (Mean data for the groups are available from the author on request.) Internal mammary artery grafting was associated with a more severe reduction in lung volumes than saphenous vein grafting and has been reported separately. " Twenty patients were transferred to hospitals close to their homes on the fifth day and this accounts for some of the missing data. The mean (SD) $\mathrm{PaO}_{2}$ was 7.37 (1.07) $\mathrm{kPa}$ on day 2 and $8.58(1 \cdot 15) \mathrm{kPa}$ on day 4 . At the time of capillary blood sampling all patients had a systolic blood pressure greater than $95 \mathrm{~mm} \mathrm{Hg}$.

\section{CHEST RADIOGRAPHS}

Radiographs taken on the first day showed areas of partial collapse or consolidation, mainly affecting the left lower lobe, in $74 \%, 74 \%$, and $75 \%$ of patients in groups 1, 2, and 3. Subsequent radiographs had improved in $24 \%, 16 \%$, and $12 \%$ and were unchanged in $52 \%, 48 \%$, and $52 \%$ of patients in the three groups $(p=0 \cdot 17)$.

\section{CHEST INFECTION}

The incidence of chest infection was $10 \%$ and occurred in four patients in group 1, two in group 2, and five patients in group $3(p>0.5)$. The patients all smoked or had only recently given up cigarettes. Mean preoperative residual volume (RV) and RV/TLC\% were higher and FER lower in these patients. After surgery FRC and $\mathrm{PaO}_{2}$ improved more slowly in patients with a chest infection.

\section{ADDITIONAL PHYSIOTHERAPY TREATMENT}

Chest physiotherapy was given to the five patients in group 3 who developed a chest infection. Preoperative $\mathrm{FEV}_{1}$ was lower in these five patients than in the remaining 32 patients in group 3 (mean (SD) $\mathrm{FEV}_{1}$ as $\%$ predicted $74.6(7.8) v 91.3(17.0) ; \mathrm{p}<0.05)$. The results of analysis for treatment differences were not influenced by exclusion of the results of these five patients. The number of self treatment sessions reported by patients in group 1 and group 2 did not differ.

No intergroup differences occurred in any of the other measures recorded.

\section{Discussion}

This study shows that a severe and persistent restrictive ventilatory defect and arterial hypoxaemia occurred after coronary artery bypass grafting. The addition of breathing exercises or incentive spirometry to a 

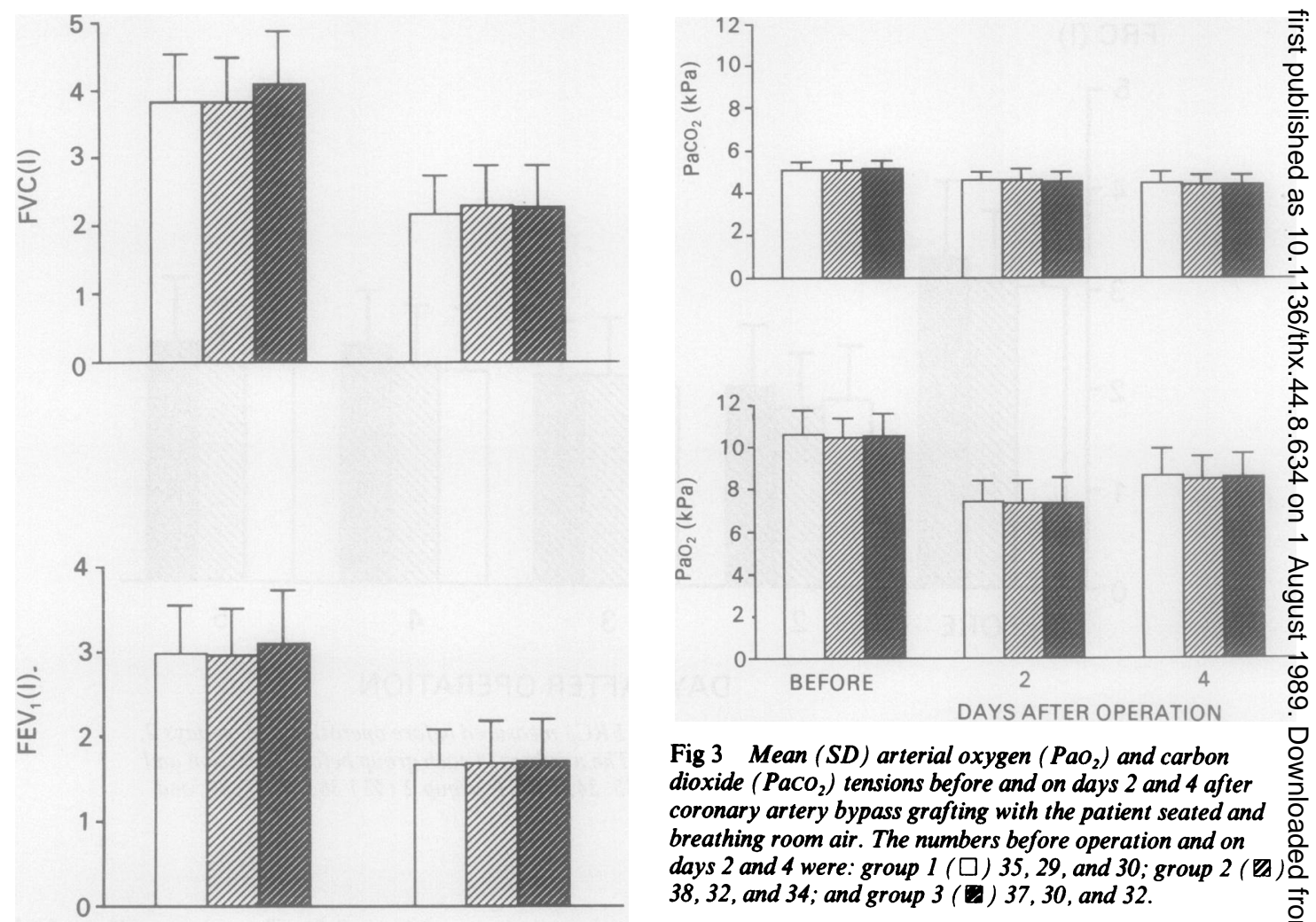

Fig 3 Mean ( $\mathrm{SD}$ ) arterial oxygen $\left(\mathrm{PaO}_{2}\right)$ and carbon dioxide $\left(\mathrm{PaCO}_{2}\right)$ tensions before and on days 2 and 4 after coronary artery bypass grafting with the patient seated and breathing room air. The numbers before operation and on

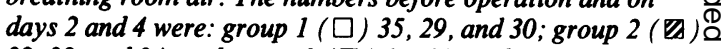
38, 32, and 34; and group 3 (3) 37, 30, and 32 .

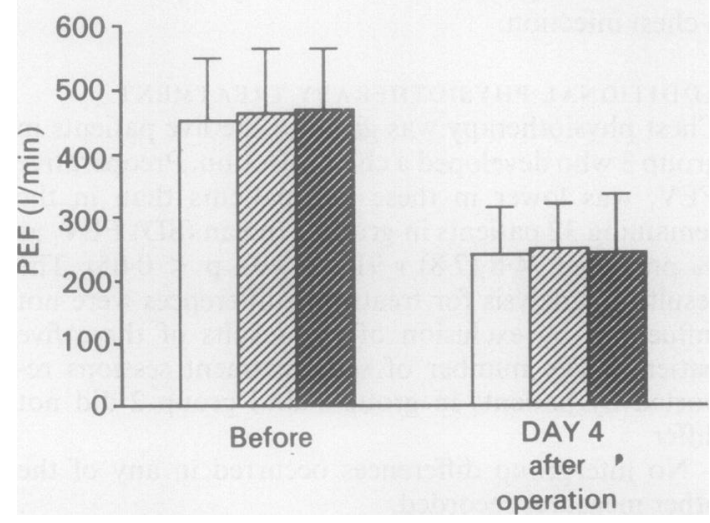

Fig 2 Mean (SD) values of forced vital capacity (FVC), $F E V_{1}$, and peak expiratory flow (PEF) before and on the fourth day after coronary artery bypass grafting with the patient seated. The numbers before and after coronary artery bypass grafting in each group were: group 1 ( $\square$ ) 35 and 33; group 2 (甘) 38 and 36; group 3 (a) 37 and 36.

regimen of early mobilisation and huffing and coughơ ing did not make postoperative lung function improve more rapidly.

We included only men in our study, to avoid the need to transport the spirometer. Differences in out:come related to gender have not been reported in other studies of postoperative physiotherapy. ${ }^{267}$ Patients with severely abnormal preoperative lung function are selected infrequently for coronary artery bypass graft-o ing and for this reason were not included in the study groups. As differences in pain tolerance related to race may influence analgesic requirements and assessment of pain, we limited our study to white people. ${ }^{12}$

The magnitude of the postoperative decrease in lung volumes and the blood gas abnormalities confirms and extends the findings of others. ${ }^{7314}$ The incidence of radiological abnormalities and their failure to improve in the early postoperative period are also in agreemen with previous findings. ${ }^{15-17}$ Measurements were now made after the fifth day because in most hospitalso physiotherapy is given for five days only and in ouro hospital many patients are transferred to other hos- $-\overrightarrow{\mathbb{D}}$ pitals on the fifth day. Recovery of lung function isD likely to be incomplete at the time of discharge from? hospital and for some time after. ${ }^{1318}$ 
Practical considerations prevented the simultaneous study of patients who were not seen at all by a physiotherapist, which would be desirable for assessing the importance of physiotherapy treatment. Patients who received only encouragement to move about and instruction in huffing and coughing recovered at the same rate as those who in addition performed breathing exercises or used an incentive spirometer. One benefit of early mobilisation is the greater increase in FRC in the erect than in the sitting posture. ${ }^{19}$ The low incidence of infection in the present study does not enable valid conclusions to be made about which physiotherapy treatment is most likely to prevent infection. Patients at increased risk can be identified on the basis of smoking history and preoperative lung function.

Our findings have important implications for the management by physiotherapists of patients undergoing coronary artery bypass grafting. Incentive spirometry and deep breathing exercises have been shown to be superior to mobilisation alone after upper abdominal surgery, ${ }^{2021}$ and our results should not be extrapolated to this group of patients or to the treatment of patients undergoing thoracotomy. We recommend that patients admitted for coronary artery bypass grafting are assessed by a physiotherapist before and after operation, taught to huff and cough effectively and encouraged to move about as soon as possible after operation. Additional physiotherapy should be considered only if the respiratory condition deteriorates or mobilisation is impossible.

We wish to thank Mr A Forsyth and Mr J R W Keates, consultant cardiothoracic surgeons for permission to study their patients. We also thank Mr D Lowe for help with the randomisation and statistical advice and Dr D Shears for her help with the analyses. This study would not have been possible without the support of the staff of the physiotherapy department. Our thanks are due to Sherwood Medical Industries for financial support. SCJ is currently funded by a Department of Health research fellowship.

\section{References}

1 Jenkins SC, Soutar SA. A survey into the use of incentive spirometry following coronary artery bypass graft surgery. Physiotherapy 1986;72:492-3.

2 Vraciu JK, Vraciu RA. Effectiveness of breathing exercises in preventing pulmonary complications following open heart surgery. Phys Ther 1977;57:1367-71.

3 Iverson LIG, Ecker RR, Fox HE, May IA. A comparative study of IPPB, the incentive spirometer, and blow bottles: the prevention of atelectasis following cardiac surgery. Ann Thorac Surg 1978;25:197-200.

4 Gale GD, Sanders DE. Incentive spirometry: its value after cardiac surgery. Can Anaesthesiol Soc J 1980; 27:475-80.

5 Oulton JL, Hobbs GM, Hicken P. Incentive breathing devices and chest physiotherapy: a controlled trial. Can J Surg 1981;24:638-40.

6 Dull JL, Dull WL. Are maximal inspiratory breathing exercises or incentive spirometry better than early mobilisation after cardiopulmonary bypass? Phys Ther 1983;63:655-9.

7 Stock MC, Downs JB, Cooper RB, et al. Comparison of continuous positive airway pressure, incentive spirometry, and conservative therapy after cardiac operations. Crit Care Med 1984;12:969-72.

8 Spiro SG, Dowdeswell IRG. Arterialized ear lobe blood samples for blood gas tensions. Br J Dis Chest 1976; 70:263-8.

9 Jenkins SC, Barnes NC, Moxham J. Evaluation of a hand-held spirometer, the Respiradyne, for the measurement of forced expiratory volume in the first second $\left(F E V_{1}\right)$ forced vital capacity (FVC) and peak expiratory flow rate (PEFR). Br J Dis Chest 1988;82: $70-5$.

10 Gaskell DV, Webber BA. The Brompton Hospital guide to chest physiotherapy. 4th ed. Oxford: Blackwell, 1980: 14-7.

11 Jenkins SC, Soutar SA, Moxham J. Lung function after coronary artery surgery using the internal mammary artery and the saphenous vein. Thorax 1989;44:209-11.

12 Woodrow KM, Friedman GD, Siegelaub MS, Collen MF. Pain tolerance: Differences according to age, sex and race. Psychosom Med 1972;34:548-56.

13 Braun SR, Birnbaum ML, Chopra PS. Pre- and postoperative pulmonary function abnormalities in coronary artery revascularization surgery. Chest 1978;73: 316-20.

14 Rea HH, Harris EA, Seelye ER, Whitlock RML, Withy SJ. The effects of cardiopulmonary bypass upon gas exchange. J Thorac Cardiovasc Surg 1978;75:104-20.

15 Good JT, Wolz JF, Anderson JT, Dreisin RB, Petty TL. The routine use of positive end-expiratory pressure after open heart surgery. Chest 1979;76:397-400.

16 Carter AR, Sostman HD, Curtis AM, Swett HA. Thoracic alterations after cardiac surgery. AJR 1983; 140:475-81.

17 Wilcox P, Baile EM, Hards J, et al. Phrenic nerve function and its relationship to atelectasis after coronary artery bypass surgery. Chest 1988;93:693-6.

18 Estenne M, Yernault JC, De Smet JM, De Troyer A. Phrenic and diaphragm function after coronary artery bypass grafting. Thorax 1985;40:293-9.

19 Blair E, Hickam JB. The effect of change in body position on lung volume and intrapulmonary gas mixing in normal subjects. J Clin Invest 1955;34:383-9.

20 Morran CG, Finlay IG, Mathieson M, McKay AJ, Wilson N, McArdie CS. Randomized controlled trial of physiotherapy for postoperative pulmonary complications. Br J Anaesth 1983;55:1113-6.

21 Celli BR, Rodriguez KS, Snider GL. A controlled trial of intermittent positive pressure breathing incentive spirometry, and deep breathing exercises in preventing pulmonary complications after abdominal surgery. Am Rev Respir Dis 1984;130:12-5.

22 Royal College of Physicians. Report of obesity. $J$ R Coll Physicians Lond 1983;17:6-65.

23 European Community for Coal and Steel Working Party. Standardization of lung function tests. Bull Eur Physiopathol Respir Clin Respir 1983;19(suppl 5):9. 